\title{
Path Planning Methods in Chemical Engineering
}

\author{
Edvards Valbahs ${ }^{1}$, Peter Grabusts ${ }^{1}$, Ilo Dreyer ${ }^{2}$ \\ ${ }^{1}$ Rezeknes Augstskola, Faculty of Engineering. \\ Address: Atbrivosanas aleja 115, Rezekne, LV-4601, Latvia \\ ${ }^{2}$ Riga Technical University, Faculty of Material Science and Applied Chemistry. \\ Address: 14/24 Azenes Street, Riga, LV-1048, Latvia
}

\begin{abstract}
Usually, when the practical motion planning and the shortest path are discoursed, mainly the limited number of tasks is observed. Almost all the tasks associated with the path from one point in 2D or 3D space to another point can be attributed to the usual issue in the practical application. Motion planning and the shortest path have vivid and indisputable importance as human activity in such areas as logistics and robotics. In our work we would like to draw particular attention to the field of application seems to be unnoticeable for the task such as motion planning and the shortest path problem. Due to quite simple examples used, we would like to show that the task of motion planning can be used for simulation and optimization of multi-staged and restricted processes which are presented in chemical engineering accordingly. In the article the simulation and optimization of three important chemicaltechnological processes for the chemical industry are discussed. The work done gave us the possibility to work out software for simulation and optimization of processes that in some cases facilitates and simplifies the work of professionals engaged in the field of chemical engineering.
\end{abstract}

Keywords: chemical industry, path planning, Travelling salesman problem, Simulated Annealing.

\section{INTRODUCTION}

The article is connected to the path planning and "Travelling salesman problem" (TSP) [2] used for decision and process automation in unusual or unexpected field of application, like chemical engineering. Practical importance of path planning is significant in transport, path planning tasks among different geographical objects. However, we would like to mention some unnoticeable cases.

Formation of genetic map - the map includes chromosomes that consist of definite marker sequence. In the given case the markers are unique DNA segments within one genome. In laboratory conditions it is possible to determine and distinguish different markers that allow forming genome maps. In order to prepare a map it is necessary to know the sequence of markers in a genome. The task of searching genome marker sequence can be modelled as TSP task. Marker sequence can be envisaged as a path along all markers. A group of researchers from National Institute of Health developed the methods and software for marker sequence searching problem [13].

Telescope orientation and searching new planets orientation of large telescopes is rather complicated and takes much energy. To decrease the period of orientation, TSP problem is applied (for example, mentioned 200 galaxies can be investigated with a telescope during one night) [15], [22].

In crystallography - positioning crystal sample in $\mathrm{x}$ ray diffractometer is a procedure happening for a long time. Due to one experiment you can include up to 30 000 different positions. When applying path planning methods, it is possible to decrease the length of experiment by $46 \%$ [14].

In electronics for example, boring holes in silicon plate, practically all the electronic silicon devices have silicon plates containing different microcircuits. Path planning application allows improving the productivity of assembly lines up to $10 \%$ [19]. One can also name the other applications in electronics: brazing silicon plates, developing computer microcircuits, searching silicon plates from defects.

Data organization in groups of elements with similar properties is the principal element in data interpretation. TSP problem was successfully adapted to solving the problem [16], [23]. TSP was successfully applied to distribute the collection of musical compositions.

German company BOWE CARDTEC develops and delivers identification and credit cards. Customers of the company book various cards. In order to make these cards, it is necessary to reconfigure equipment from one working task to the other working task and this is rather prolonged operation. To improve this 
process, path planning methods are applied and the entire process was modelled as path planning problem. Working tasks were presented like cities, but the time required to make the reconfigurations was presented in a kind of distance among the cities. Path planning problem gives the possibility to improve the productivity up to $65 \%$ [10].

Our previous article was dedicated to mobile agent that moved across 2D closed premises and path planning was successfully applied [12]. Now we would like to show that path planning can be successfully applied for some chemical engineering processes. More detailed motivations and goals are described in the next section of our article.

\section{GOALS}

The aim of the article is to describe some chemical engineering approaches to the production of substances as multistaged process. The processes have to be fulfilled in succession, controlling the considerable parameters of processes. The quality and quantity of selected product depends on sequence of the process stages and control of characteristic parameters a great deal. Actually in chemical engineering one or several alternative stages can be selected for any stage of process which deals with substances production. Alternative stages differ from each other with completely definite parameters set which characterizes selected stage. Thus, according to previously mentioned technological processes can be characterized as follows:

- certain branching - each alternative branch can be viewed as alternative path;

- selection among alternative stages - it is possible to select one solution from several paths;

- $\quad$ stage characterized with parameters - there is a possibility to characterize alternative stages with definite parameters set.

Taking into account the above mentioned marks, it can be confirmed that chemical engineering processes are characterized with a tree or graph (i.e. the data structure in a kind of a tree or graph). Therefore, chemical engineering processes can be viewed within path planning task. Our aim is to discuss chemical engineering processes as graph-type data structure for the following substances or groups of substances (that allows programming decision-making and process analysis):

- Borane - it can be used in the area of highenergy fuels for jet planes and rockets. The thermal decomposition of diborane $\left(\mathrm{B}_{2} \mathrm{H}_{6}\right)$ has been used to produce coatings of pure elementary boron for neutron-detecting devices and applications requiring hard, corrosionresistant surfaces. Boranes can be used as vulcanizing agents for natural and synthetic rubbers, and are particularly effective in the production of silicone rubbers [6], [11], [17];

- Malonic acid derivatives - widely used in biochemistry and biology [4], [24].

The above mentioned compounds have been selected, for there are a lot of scientific sources that describe the obtaining of given compounds and in the same time describe all the possible alternatives as well.

\section{ASSUMPTIONS}

To fulfil the previously mentioned goals, it is necessary to reflect the processes of obtaining borane and aluminium oxide in a kind of graphs. The structure of graphs allows performing further analysis of processes. Correlation among the substances involved in the process and process stage both can be presented as a table. The process of obtaining borane is described in Table 1.

TABLE 1.

BORANE OBTAINING PROCESS

\begin{tabular}{|c|c|c|c|c|}
\hline \multicolumn{2}{|c|}{$\begin{array}{c}\text { SUBSTANCES BEFORE } \\
\text { CONVERSION (OR } \\
\text { CURRENT STAGE OF THE } \\
\text { PROCESS) }\end{array}$} & \multirow{2}{*}{ 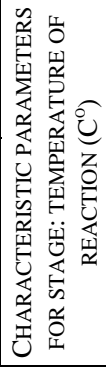 } & \multicolumn{2}{|c|}{ PRODUCT OF STAGE } \\
\hline 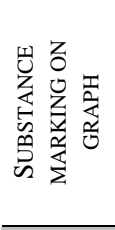 & $\begin{array}{c}\text { NAME OF } \\
\text { SUBSTANCE } \\
\text { AND FORMULA }\end{array}$ & & $\begin{array}{l}\text { NAME OF } \\
\text { SUBSTANCE } \\
\text { AND } \\
\text { FORMULA }\end{array}$ & 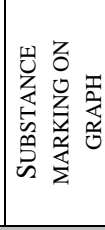 \\
\hline \multirow[t]{2}{*}{1} & $\begin{array}{l}\text { B4H10 - } \\
\text { tetraborane }\end{array}$ & 200 & $\begin{array}{l}\text { B5H9 - } \\
\text { pentaborane }\end{array}$ & 2 \\
\hline & & 60 & $\begin{array}{l}\text { B5H11 - } \\
\text { pentaborane }\end{array}$ & 5 \\
\hline 2 & $\begin{array}{l}\text { B5H9 - } \\
\text { pentaborane }\end{array}$ & - & - & - \\
\hline \multirow[t]{4}{*}{3} & $\begin{array}{l}\text { B2H6 - } \\
\text { diborane }\end{array}$ & 180 & $\begin{array}{l}\text { B4H10 - } \\
\text { tetraborane }\end{array}$ & 1 \\
\hline & & 130 & $\begin{array}{l}\text { B5H9 - } \\
\text { pentaborane }\end{array}$ & 2 \\
\hline & & 280 & $\begin{array}{l}\text { B10H14 - } \\
\text { decaborane }\end{array}$ & 4 \\
\hline & & 115 & $\begin{array}{l}\text { B5H11 - } \\
\text { pentaborane }\end{array}$ & 5 \\
\hline 4 & $\begin{array}{l}\text { B10H14 - } \\
\text { decaborane }\end{array}$ & 95 & $\begin{array}{l}\text { B4H10 - } \\
\text { tetraborane }\end{array}$ & 1 \\
\hline \multirow[t]{2}{*}{5} & $\begin{array}{l}\text { B5H11 - } \\
\text { pentaborane }\end{array}$ & 25 & $\begin{array}{l}\text { B10H14 - } \\
\text { decaborane }\end{array}$ & 4 \\
\hline & & 100 & $\begin{array}{l}\text { B4H10 - } \\
\text { tetraborane }\end{array}$ & 1 \\
\hline
\end{tabular}

For example, the two substances (two different pentaboranes) can be obtained from tetraborane (Table 1 ), but at different temperatures (in this case the temperature is an important parameter of current stage). Moreover, tetraborane can be obtained either from decaborane, pentaborane or diborane (Table 1). 
The graph (Fig. 1) related to borane obtaining process (Table 1) is built up, where graph nodes are substances and graph edges are the temperature in each stage. Each stage has its own weight and is relevant to the temperature.

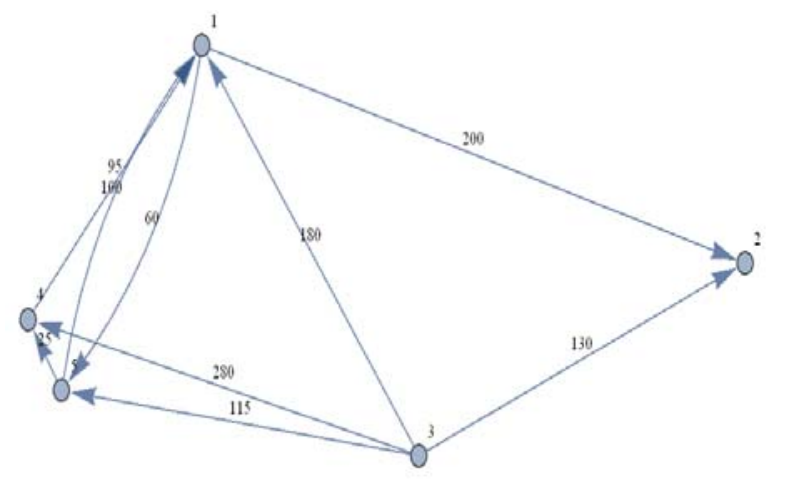

Fig. 1. Graph for borane obtaining process

It is needed to mention that temperature plays a very important role in chemical technology. Actually, all chemical processes are taking place at definite temperature, which stimulates or suppresses the processes. It means that the temperature within the process should be sustained, i.e. supplied with additional amount of warmth or it can be removed. All manipulations with amount of warmth are directly related to power consumption. Actually, in chemical technology with amount of warmth energy efficiency of process can be characterized. Chemical engineer, designing technological process, is always interested in decreasing heat consumption, i.e. selecting a process where the final product is obtained with lower consumption of heat as far as possible, increasing energy efficiency of obtaining substance accordingly.

As to malonic acid derivatives, the design principle of previously mentioned graph remains with its own specific features, related to stage parameters, i.e., outcome of reaction Table 2 .

TABLE 2.

MALONIC ACID DERIVATIVES OBTAINING PROCESS

\begin{tabular}{|c|c|c|}
\hline $\begin{array}{c}\text { SUBSTANCES BEFORE } \\
\text { CONVERSION (OR } \\
\text { CURRENT STAGE OF } \\
\text { THE PROCESS) }\end{array}$ & 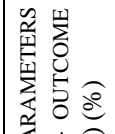 & PRODUCT OF STAGE \\
\hline $\begin{array}{c}\text { SUBSTANCE MARKING } \\
\text { ON GRAPH }\end{array}$ & 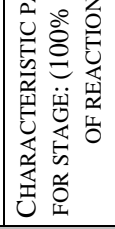 & $\begin{array}{c}\text { SUBSTANCE MARKING ON } \\
\text { GRAPH }\end{array}$ \\
\hline A1 & 40 & A2 \\
\hline A2 & 35 & A3 \\
\hline A3 & 31 & B3 \\
\hline B1 & 55 & B2 \\
\hline
\end{tabular}

\begin{tabular}{lll} 
B2 & 30 & B3 \\
B3 & 10 & C2 \\
& 30 & D5 \\
C1 & 2 & D4 \\
C2 & 50 & C1 \\
D1 & 24 & D2 \\
D2 & 45 & D3 \\
D3 & 4 & D4 \\
D4 & 33 & D5 \\
D5 & 5 & D6 \\
D6 & - & - \\
\hline
\end{tabular}

Table 3 shows the names of malonic acid derivatives in accordance with the names of vertexes in the graph (Fig. 2).

TABLE 3.

NAMES OF MALONIC ACID DERIVATIVES IN THE GRAPH

\begin{tabular}{|c|c|}
\hline $\begin{array}{c}\text { SUBSTANCE } \\
\text { MARKING ON } \\
\text { GRAPH }\end{array}$ & $\begin{array}{l}\text { NAME OF SUBSTANCE AND FORMULA (WHERE R } \\
\text { IS ALKANE DERIVATIVE) }\end{array}$ \\
\hline A1 & di(R) 2-((R)oxymethylidene)propanedioate \\
\hline A2 & R 4-hydroxy-2-methylpyrimidine-5-carboxylate \\
\hline A3 & R 4-chloro-2-methylpyrimidine-5-carboxylate \\
\hline B1 & 2-((R)oxymethylidene)propanedinitrile \\
\hline B2 & $\begin{array}{l}\text { R (2E)-3-[(Z)-(1-aminoethylidene)amino]-2- } \\
\text { cyanoprop-2-enoate }\end{array}$ \\
\hline B3 & R 4-amino-2-methylpyrimidine-5-carboxylate \\
\hline $\mathrm{C} 1$ & 4-amino-2-methylpyrimidine-5-carboxamide \\
\hline $\mathrm{C} 2$ & 4-amino-2-methylpyrimidine-5-carbonitrile \\
\hline D1 & $\begin{array}{l}\text { 4,6-dihydroxy-2-methylpyrimidine-5- } \\
\text { carboxamide }\end{array}$ \\
\hline D2 & 4,6-dichloro-2-methylpyrimidine-5-carbonitrile \\
\hline D3 & $\begin{array}{l}\text { 4-amino-6-chloro-2-methylpyrimidine-5- } \\
\text { carbonitrile }\end{array}$ \\
\hline D4 & 5-(aminomethyl)-2-methylpyrimidin-4-amine \\
\hline D5 & (4-amino-2-methylpyrimidin-5-yl)methanol \\
\hline D6 & 5-(chloromethyl)-2-methylpyrimidin-4-amine \\
\hline
\end{tabular}

Weights of edges at the graph are proportional to the products of the reaction (Fig. 2).

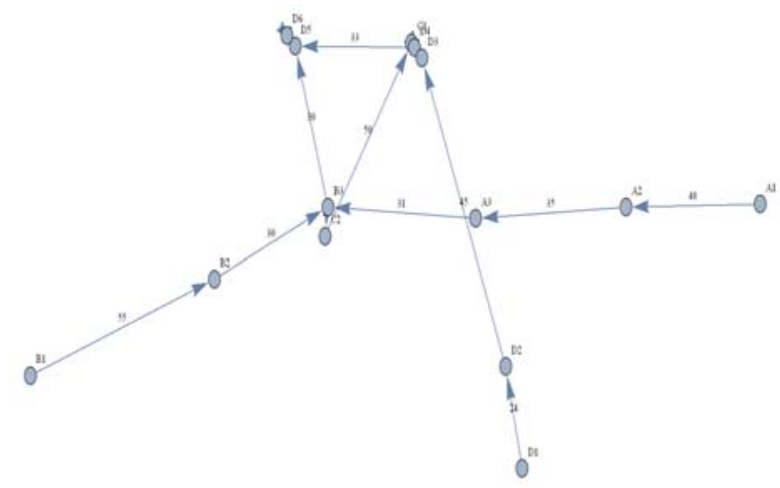

Fig. 2. Graph for malonic acid derivatives obtaining process 
Outcome of reaction is amount of product obtained as a result of chemical process/reaction (it is a very important parameter in chemical engineering) and it is always less than 100 per cent (it characterizes the effectiveness of reaction). It can be seen that the higher outcome of the stage, the better result is achieved according to the parameter. But we should take into consideration that for path planning it is better to have a total distance which must be the smallest as far as possible. Thus, admittedly, value of edges in the given case is difference between 100 per cent and outcome of current stage.

\section{RESULTS}

Discussing processes of forming borane and aluminium oxide, it can be assumed that all stages take one and the same prolongation of time. If time of reaction is unchangeable in all stages, then difference of stages including amount of warmth will depend only on temperature. It is possible to find the most energy-efficient path studying the graph of process and looking for the shortest path from start vertex to final one. Fig. 3 shows the most energy-efficient path from diborane (vertex 3) to decaborane (vertex 4) in the process of obtaining boranes (Table 1).

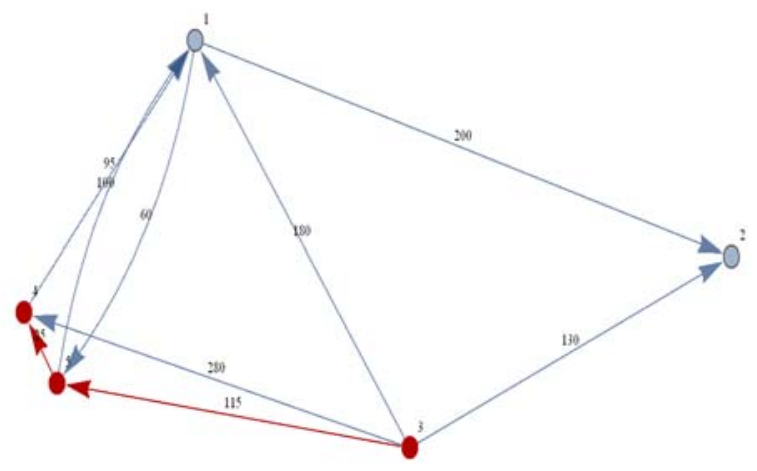

Fig. 3. The most energy-efficient path from diborane to decaborane

The production of decaborane (vertex 1) from tetraborane is more beneficial in comparison with the production from diborane (it can be vividly seen from Fig. 4 that the total length of the path is shorter compared with the previous example).

If it is necessary to obtain the group of substances tetraborane, pentaborane (vertex 5) and decaborane as energy-efficient path, it is needed to consider the part of the graph which does not contain vertex 2 (Fig. 5).

The shortest path among all the vertexes in the graph can be obtained as a part of TSP, i.e. Simulated annealing (SA) can be used to find the energyefficient path in the graph [1], [3], [5], [7], [18], [20], [21].

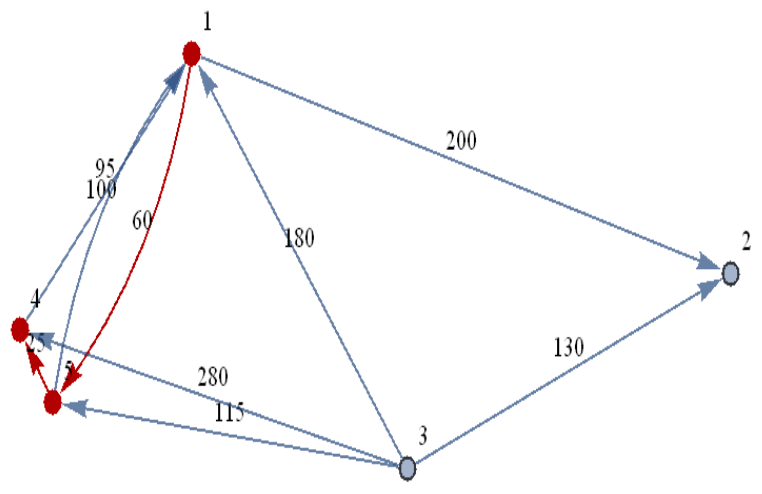

Fig. 4. The most energy-efficient path from diborane to decaborane

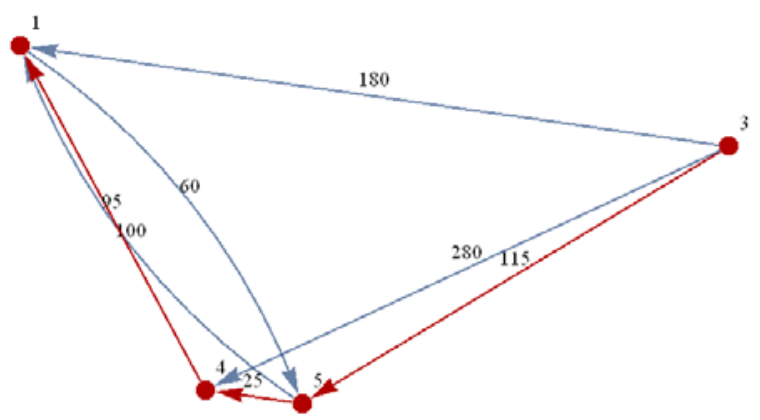

Fig. 5. The graph presents the part of boranes obtaining process with the shortest path among all the vertexes

Outcome of reaction and temperature are of great practical importance. Outcome of reaction shows what amount of substance of the initial mixture of substances is obtained at the end of conversion to the product of chemical reaction. Outcome of reaction reveals the efficiency of substances consumption in chemical processes. Therefore, it can be confirmed that the given parameter determines exactly economic indicators of the processes (as the substance can be also bought without production, and it is necessary to decide either it should be bought or produced and it will be reflected in terms of money). For stages of the process on obtaining derivatives of malonic acid different alternatives can be viewed which considerably differ by outcome of reaction, but not by temperature. Let us look at the graph for malonic acid derivatives obtaining process. A graph structure can be extended by assigning a weight to each edge as difference between 100 per cent and outcome of reaction, and by this way the shortest path is explained to be looked for in the graph. As the shortest path is searched the less the edge is the better result is. And on the contrary, it is better when the outcome of reaction reaches to 100 per cent. In order to apply path planning methods, in this case the weight of edge should be calculated as difference between 100 per cent and outcome of reaction, 100 per cent being theoretical maximal value of outcome of reaction. The 
shortest path from di(R) 2-((R)oxymethylidene) propanedioate (vertex $\mathrm{A} 1$ and where $\mathrm{R}$ is alkane derivative) to 5-(chloromethyl)-2-methylpyrimidin-4amine (vertex D6) for malonic acid derivatives obtaining process (Table 2) is presented in Fig. 6.

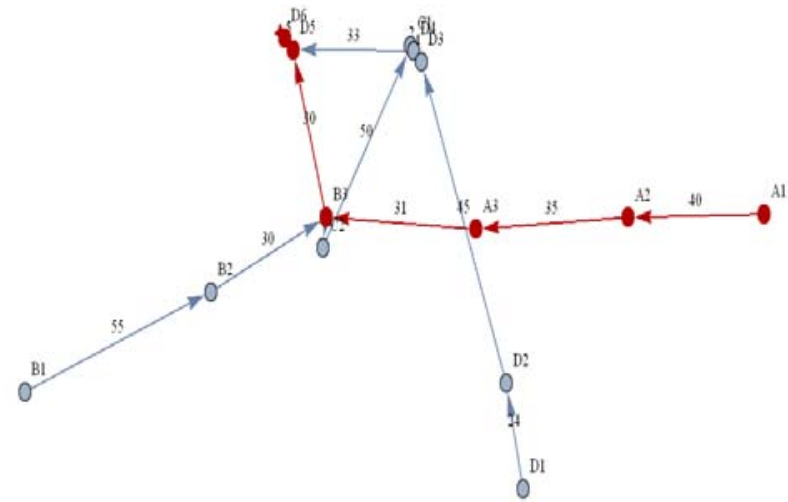

Fig. 6. The shortest path from di(R) 2-((R)oxymethylidene) propanedioate (where $\mathrm{R}$ is alkane derivative) to 5-(chloromethyl)-2methylpyrimidin-4-amine (total path length is 141 per cent)

The shortest path from 2-((R)oxymethylidene) propanedinitrile (vertex B1 and where $\mathrm{R}$ is alkane derivative) to 5-(chloromethyl)-2-methylpyrimidin-4amine (vertex D6) is depicted Fig. 7.

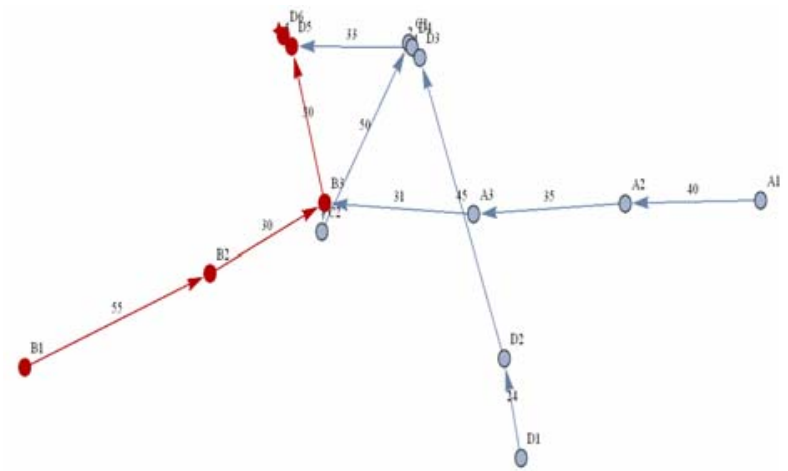

Fig. 7. The shortest path from 2-((R)oxymethylidene) propanedinitrile (where $\mathrm{R}$ is alkane derivative) to 5-(chloromethyl)2-methylpyrimidin-4-amine (total path length is 120 per cent)

Fig. 8 shows the shortest and most efficient path for the outcome of the reaction (compared with the previously mentioned cases) from 4,6-dihydroxy-2methylpyrimidine-5-carboxamide (vertex D1) to 5(chloromethyl)-2-methylpyrimidin-4-amine (vertex D6) .

The shortest path from 4,6-dihydroxy-2methylpyrimidine-5-carboxamide (vertex D1) to 5(aminomethyl)-2-methylpyrimidin-4-amine (vertex D4) is shown in Fig. 9.

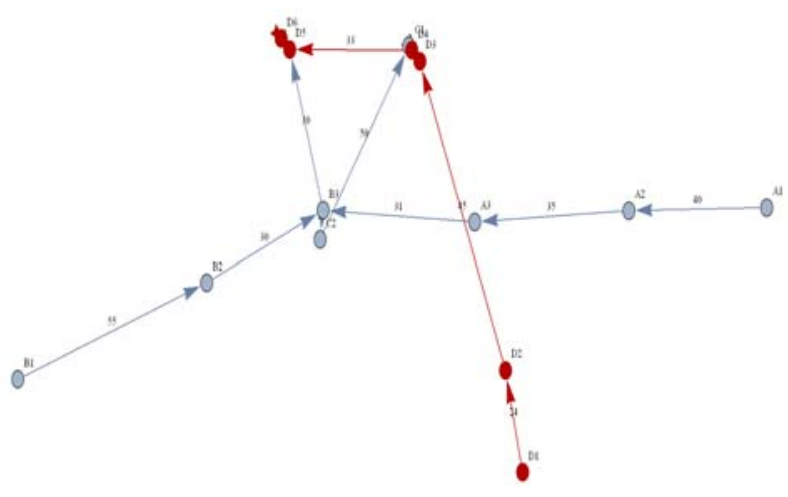

Fig. 8. The shortest path from 4,6-dihydroxy-2methylpyrimidine-5-carboxamide to 5-(chloromethyl)-2methylpyrimidin-4-amine (total path length is 111 per cent)

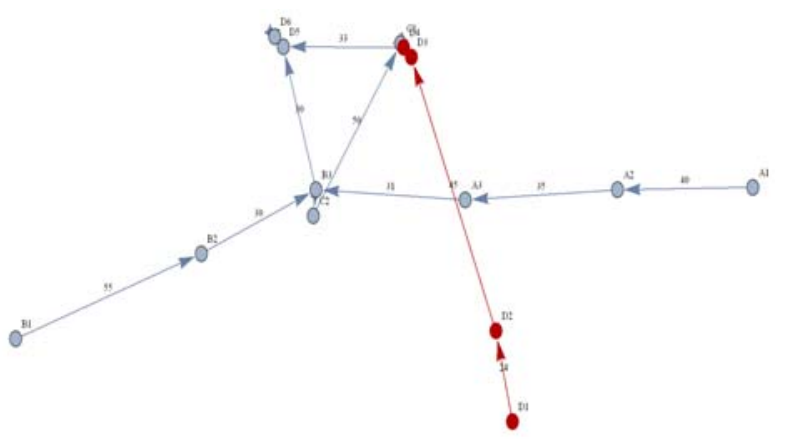

Fig. 9. The shortest path from 4,6-dihydroxy-2methylpyrimidine-5-carboxamide to 5-(aminomethyl)-2methylpyrimidin-4-amine (total path length is 73 per cent)

The shortest and most efficient path for the outcome of the reaction (compared with the previously mentioned cases) from $\mathrm{R}$ 4-amino-2methylpyrimidine-5-carboxylate (vertex B3 and where $\mathrm{R}$ is alkane derivative) to 5-(aminomethyl)-2methylpyrimidin-4-amine (vertex D4) is depicted in Fig. 10.

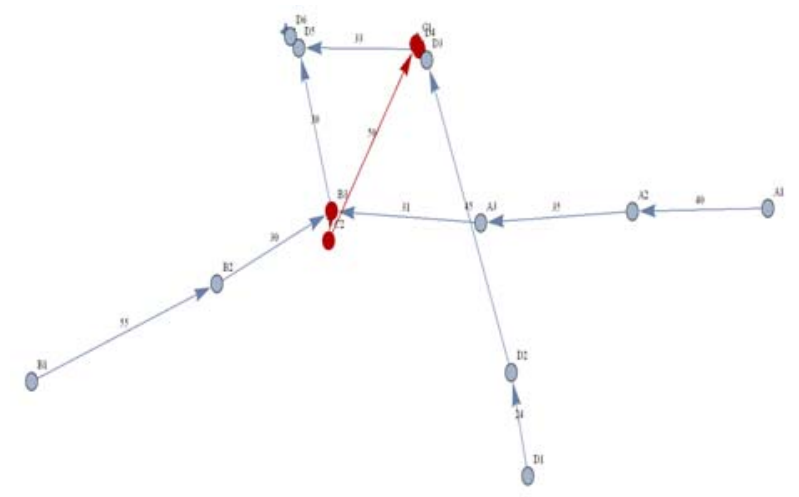

Fig. 10. The shortest path from R 4-amino-2-methylpyrimidine-5carboxylate (where $\mathrm{R}$ is alkane derivative) to 5-(aminomethyl)-2methylpyrimidin-4-amine (total path length is 62 per cent) 


\section{CONCLUSION}

Submitted examples show that TSP and path planning can be successfully used in automatizing the analysis and decision-making for borane, malonic acid derivatives and other processes for production of chemical substances. Automated decision-making and analysis give the possibility to facilitate the design process. Optimal conditions of the process in selected technological processes are rather easy to be found without using any software, which are done by engineers in industry and searching these optimal conditions extends the design process. If a process contains more alternative stages than those in the selected processes, the design process is expected to be protracted, which will also increase the price of technological process implementation. Design practices can be improved even with a partial atomization of the designing process.

We would like to mention that many linked substances (vertexes) are involved in chemical engineering processes. The methods of obtaining substances often change (e.g. production of bioactive substances). Chemical engineering processes are dynamic systems that change from time to time. A lot a scientific researcher is conducted and as a result known products can be obtained in more efficient processes in comparison with technologies that are well know nowadays.

Working out new technologies it is necessary to take into a count the competitive period of time for chemical technology or process developed. It is needed to know the parts of technology that will have to be changed in the nearest future in order to keep the competitiveness of technology. Observing technological process from the point of view of such approach gives the possibility to make necessary changes more efficiently [8]. Taking in to account the previous mentioned it is impossible to find the shortest path (more effective solution) by heart in systems or technology said [9]. In addition, we would like to mention the example of robot scientist Eve which was developed by the researches and engineers from Cambridge and the University of Manchester. Robot Eve is successfully involved in the developing process of new medicines. This work is an excellent combination of robotics, artificial intelligence and pharmacology [25]. We would like to demonstrate that currently, artificial intelligence methods can contribute a considerable for chemical engineering.

The programme product developed in the context of this work simplifies the process of designing for borane and malonic acid derivatives obtaining processes. It is easy to track optimization process using the TSP and path planning because all the selected examples of chemical engineering processes in this article are simple. In our views, TSP, path planning algorithms and methods can be successfully applied to other chemical engineering processes optimization tasks with much more complexity (with the large number of alternative stages and other wider group of parameters, i.e. not only for temperature).

As it was mentioned in the Introduction, TSP and the shortest path problem have specific field of application which seems to be unnoticeable:

- formation of genetic map;

- telescope orientation and searching new planets;

- positioning crystal sample in x-ray diffractometer;

- $\quad$ boring holes in silicon plate;

- data organization in groups of elements;

- decreasing wallpaper strips;

- $\quad$ cutting templates in glass production;

- $\quad$ investigating the problem in evolution process.

We suppose that the mentioned list can be added with the new application of TSP and the shortest path problem, i.e. application in chemical engineering. As regards the chemical engineering, the implementation of TSP and the shortest path problem give the opportunity of developing new methodology for chemical engineering.

\section{REFERENCES}

[1] R. Azencott, Simulated annealing: parallelization techniques. John Wiley and Sons, 1992.

[2] W.J. Cook, In Pursuit of the Traveling Salesman. Princeton: Princeton University Press, USA, 2011.

[3] D. D. Davendra, Traveling Salesman Problem, Theory and Applications. Rijeka: InTech, 2010.

[4] P. Patnaik, Handbook of Inorganic Chemicals. McGraw-Hill, 2003.

[5] E. Aarts and J. Korst, Simulated annealing and Boltzmann machines: A stochastic approach to combinatorial optimization and neural computing. John Wiley and Sons, 1989.

[6] N.N. Greenwood and A. Earnshaw, Chemistry of the Elements. $2^{\text {nd }}$ ed., Elsevier - Butterworth-Heinemann, 1997.

[7] R. H. J. M. Otten and L. P. P. P. Ginneken, The Annealing Algorithm. Kluwer Academic Publishers, 1989.

[8] G. Towler and R. Sinnott, Chemical Engineering Design, Second Edition: Principles, Practice and Economics of Plant and Process Design. $2^{\text {nd }}$ ed., Butterworth-Heinemann, 2012.

[9] D. Voet, J.G. Voet and C.W. Pratt, Principles of Biochemistry. $4^{\text {th }}$ ed., John Wiley \& Sons, Inc., 2012.

[10] D.L. Applegate, R. Bixby, V. Chvátal and W. Cook, The Traveling Salesman Problem: A Computational Study. Princeton University Press, 2006.

[11] F. A. Cotton, G. Wilkinson, C.A. Murillo and M. Bochmann, M, Advanced Inorganic Chemistry. 6th ed., John Wiley \& Sons: New York, 1999.

[12] E. Valbahs and P. Grabusts, Path Planning Using Non Euclidean Metric, 8th EUROSIM Congress on Modelling and Simulation EUROSIM2013, Cardiff, Wales, United Kingdom, 10-12 September, 173-178, 2013.

[13] R. Agarwala, D.L. Applegate, D. Maglott, G.D. Schuler and A.A. Schäffer, "A Fast and Scalable Radiation Hybrid Map Construction and Integration Strategy," Genome Research, 10, 350-364, 2000.

[14] R.G. Bland and D.F. Shallcross, "Large traveling salesman problems arising from experiments in x-ray crystallography," Operations Research Letters, 8, 123-133, 1989.

[15] S. Carlson, "The Amateur Scientist: Telescopes and a problem for traveling salesmen in space," Scientific American, 276, 121 - 123, 1997. 
[16] S. Climer and W. Zhang, "Rearrangement clustering: Pitfalls, remedies, and applications,” Journal of Machine Learning Research, 7, 919-943,2006.

[17] M.A. Fox and K. Wade, "Evolving patterns in boron cluster chemistry,” Pure Appl. Chem., 75 (9), 1315-1323, 2003.

[18] V. Granville, M. Krivanek and J-P. Rasson, "Simulated annealing: A proof of convergence," IEEE Transactions on Pattern Analysis and Machine Intelligence, 16(6), 652-656, 1994.

[19] M. Grötschel, M. Jünger and G. Reinelt, "Optimal Control of Plotting and Drilling Machines: A Case Study,” Zeitschrift für Operations Research, 35(1), 61-84, 1991.

[20] L. Ingber, "Simulated annealing: Practice versus theory," Math. Comput. Modelling, 18, 29-57, 1993.

[21] S. Kirkpatrick, C.D. Gelatt and M.P. Vecchi, "Optimization by Simulated Annealing,” Science, vol. 220, 671-680, 1983.
[22] E. Kolemen and N.J. Kasdin, "Optimal Trajectory Control of an Occulter-Based Planet-Finding Telescope," Advances in Astronautical Sciences, AAS 07-037, Vol. 128, 215- 233, 2007.

[23] J.K. Lenstra, "Clustering a data array and the traveling salesman problem,” Operations Research, 22, 413-414, 1974.

[24] N. Weiner, "Malonic acid. Organic Syntheses," Coll. Vol. 2, 376, 1943.

[25] "Robot scientist Eve will allow scientists to save time and money in the development of new drugs," [Online]. Available:http://www.supersait.org/robot-scientist-eve-willallow-scientists-to-save-time-and-money-in-the-

development-of-new-drugs.html\#more-410 [Accessed: February 23, 2015]. 